\title{
PREDICTIVE STOP-LOSS PREMIUMS
}

\author{
BY WERNER HÜRLIMANN ${ }^{1}$ \\ Switzerland
}

\begin{abstract}
Based on a representation of the aggregate claims random variable as linear combination of counting random variables, a linear multivariate Bayesian model of risk theory is defined. In case of the classical risk theoretical assumptions, that is conditional Poisson likelihood counting variates and Gamma structural density, the model is shown to identify with a Bayesian version of the collective model of risk theory. An interesting multivariate credibility formula for the predictive mean is derived. A new type of recursive algorithm, called three-stage nested recursive scheme, allows to evaluate the predictive density and associated predictive stop-loss premiums in an effective way.
\end{abstract}

\section{KEYWORDS}

Aggregate claims; stop-loss; Bayesian modelling; collective risk theory; recursive algorithm.

\section{INTRODUCTION}

It is well-known that the fluctuation of basic probabilities in a portfolio of risks plays an important role. Early work has been done by AMMETER (1948/1949) and the subject is emphasized in BEARD et al. (1984). The actuarial literature devoted to stochastic variation of mortality and other types of mortality variation is relatively scarce. A review of known studies is given by WoLTHUIS and VAN HoEK (1986), Section 7. More recent work includes NoRBERG (1987) and KLUGMAN (1989). Random variation in uncertain payments taking into account other sources of variation is discussed in DE JONG (1983).

In life insurance the observed mortality experience of a group contract may deviate considerably from the expected mortality given by a life table. This means that the expected value of aggregate claims may also deviate considerably from the value obtained from a life table. Since priorities of stop-loss contracts are usually expressed as percentages of the mean aggregate claims and stop-loss premiums are very sensitive with respect to this quantity, it follows that the impact of the variation in basic probabilities on the aggregate

1 This work was originally presented at the meeting on Risk Theory in Oberwolfach, September 16-22, 1990. 
claims distribution and derived quantities such as mean, standard deviation and stop-loss premiums may be important. When tariffing stop-loss contracts and in order to avoid mismatches in priorities, the estimation of the mean aggregate claims needs special attention. In this paper an attempt is made to take into account claims experience when evaluating aggregate claims distributions and related stop-loss premiums. The considered Bayesian (analytical) model is derived from the well-known standard tools of Decision Theory. The mathematical steps involved in the construction of a feasible computational algorithm are however rather formidable and are based on previous results of the author. Analogous results for alternate Bayesian models chosen from among other natural conjugate families may be possible but are not considered in this paper. It is also most desirable to develop distribution-free formulas following eventually new paradigms as suggested by JEWELL (1990). In the following let us give a more detailed outline of the paper with its main results.

In Section 2 the random variable of aggregate claims associated to a portfolio of risks is viewed as a linear combination of counting random variables for which there exist computational algorithms to evaluate the corresponding distribution function (see HÚRLIMANN (1990a)). It is assumed that risk units produce claims of known amount and that the probability of occurrence of a given claim is an unknown following some structural density. Based on these assumptions a linear multivariate Bayesian model of risk theory is defined. If one adds further the natural model assumptions used in life and general insurance, one obtains what we call linear multivariate Poisson Gamma Bayesian model of risk theory. In the present work only this special model is studied. To illustrate the results of the paper a simple life insurance example is presented and used throughout.

In Section 3 a link to classical collective risk theory is given. It is shown that the linear multivariate Poisson Gamma Bayesian model coincides with a well-defined Bayesian version of the collective model of risk theory.

The needed Bayesian formulas to perform later on effective stop-loss premium calculations are dereived in Section 4. As main results we obtain an appealing multivariate credibility formula for the predictive mean of aggregate claims and an analytical representation of the predictive density defined earlier by JEWELL (1974).

Using two recursive algorithms to evaluate the probability distribution function of a linear combination of independent random variables first derived in HÜrlimanN (1990a) and reviewed in the Appendix, we derive in Section 5 a three-stage nested recursive scheme to evaluate the predictive density of our Bayesian model. Previous numerical algorithms for aggregate claims probability models involved so far only one and two-stage nested recursive calculations as can be seen from the last advances in the insurance field by PANJER (1981), De PRIL (1986/1989) and HÜRLIMANN (1990a/b).

Finally in Section 6 a numerical example illustrates the important impact claims experience may have on mean, cumulative probability and stop-loss premiums evaluated in a Bayesian framework. 


\section{A LINEAR MULTIVARIATE BAYESIAN MODEL OF RISK THEORY}

For the purpose of rating stop-loss and other non-proportional reinsurance contracts, we consider the random variable of aggregate claims which is associated to a given portfolio of risks. Assume that the risks in question are subject to the following risk classification system:

- each risk unit ( $=$ individual policy exposed to risk) can produce a claim of a known amount $m_{k}, k=1, \ldots, r$, where $r$ is the number of possible amounts

- a claim is characterised by an unknown probability of occurrence $\theta_{i}$, $i=1, \ldots, \mathrm{s}$, where $s$ is the number of different probabilities of occurrence

\section{Example 2.1}

Consider a life insurance portfolio subject to the risk of death and/or disability. Each life consists of at most three insurable risk units, two for death and one for disability, producing claims whose amounts at risk can be evaluated using computer programs. In pension insurance they are routinely calculated using well-known formulas (e.g. BERTRAM and FEILMEIER (1987), Section 3.2.1, pp. 61-64). Given a life aged $x$, a claim for the risk of death may occur with the unknown probability $q_{x}^{a}$ if the insured dies as active member, or it may occur with the unknown probability $q_{x}^{i}$ if the insured dies as disabled member, both with different amounts at risk. A claim for the risk of disability may occur with the unknown probability $i_{x}$. It is straightforward to obtain the above risk classification system by renaming the variables appropriately.

Given the above risk classification system, let us consider the following mathematical model of aggregate claims. Let $X_{k i}$ be the random variable counting the number of claims of amount at risk $m_{k}$ with probability of occurrence $\theta_{i}$. Then the random variable representing the aggregate claims is given by

$$
X=\sum_{k=1}^{r} m_{k} \sum_{i=1}^{s} X_{k i}
$$

The uncertainty about $\theta_{i}$ is modelled by a prior or structural density denoted $u_{i}\left(\theta_{i}\right), i=1, \ldots, s$. We assume independence between the $\theta_{i}$ 's. Therefore the structural density of the parameter vector $\boldsymbol{\theta}=\left(\theta_{1}, \ldots, \theta_{s}\right)$ is given by

$$
u(\theta)=\prod_{i=1}^{s} u_{i}\left(\theta_{i}\right)
$$

Specifying different assumptions on the conditional probabilities $\operatorname{Pr}\left(X_{k i}=j \mid \theta_{i}\right)$ that $j$ claims of amount $m_{k}$ (given $\theta_{i}$ ) occur, and on the structural densities $u_{i}\left(\theta_{i}\right)$, it is possible to obtain different Bayesian models to describe and analyze the aggregate claims random variable $X$. The following natural model assumptions are widely used in life as well as in general insurance: 
(I) Conditionally on $\theta$ the random variables $X_{k i}$ are independent and depend upon $\theta_{i}$ through the Poisson likelihood

$$
\operatorname{Pr}\left(X_{k i}=j \mid \theta_{i}\right)=\operatorname{Po}\left(j ; \theta_{i} n_{k i}\right)=e^{-\theta_{i} n_{k i}} \frac{\left(\theta_{i} n_{k i}\right)^{j}}{j !}, \quad j=0,1,2, \ldots,
$$

where $n_{k i}$ is the number of risk units with amount at risk $m_{k}$ and probability of occurrence $\theta_{i}$.

(II) The structural densities $u_{i}\left(\theta_{i}\right)$ are Gamma densities given by

$$
u_{i}\left(\theta_{i}\right)=\operatorname{Gamma}\left(\theta_{i} ; \alpha_{i}, \beta_{i}\right)=\frac{\beta_{i}^{\alpha_{i}} \theta_{i}^{\alpha_{i}-1} e^{-\beta_{i} \theta_{i}}}{\Gamma\left(\alpha_{i}\right)}, i=1,2, \ldots, s, \alpha_{i}, \beta_{i}>0 \text {. }
$$

At this stage it is possible to get formulas for the moments of $X$. The expected value, which will be needed later on, is calculated as follows:

$$
\begin{aligned}
E[X] & =\sum_{k=1}^{r} m_{k} \sum_{i=1}^{s} E\left[X_{k i}\right]=\sum_{k=1}^{r} m_{k} \sum_{i=1}^{s} E_{0_{i}}\left[E\left[X_{k i} \mid \theta_{i}\right]\right] \\
& =\sum_{i=1}^{s} \frac{\alpha_{i}}{\beta_{i}} \sum_{k=1}^{r} m_{k} n_{k i}
\end{aligned}
$$

The results of the present paper will be illustrated numerically at the following simple life insurance portfolio.

\section{Example 2.2}

Given is a portfolio of 1500 active persons insured against the risk of death. It is divided into $s=3$ age classes corresponding to the approximate ages $x=30,40,50$ with probabilities of death $q_{30}=0.00051, q_{40}=0.00114$, $q_{50}=0.00344$ borrowed from the EVK80 table, which is the life table of the "Eidgenössiche Versicherungskasse" used in Swiss pension insurance for rating risk of death and disability. Each age class is subdivided into $r=5$ risk sums subclasses with lump sums $500^{\prime} 000,1^{\prime} 000^{\prime} 000,1^{\prime} 500^{\prime} 000,2^{\prime} 000^{\prime} 000$ and $2^{\prime} 500^{\prime} 000$. Choosing a risk unit of $\Delta=500^{\prime} 000$, this means that $m_{k}=k$, $k=1,2, \ldots, 5$. The number $n_{k i}$ of persons in each of the 15 subclasses is as follows:

\begin{tabular}{l|ccccc}
\hline \hline & \multicolumn{5}{c}{$n_{k i}$} \\
\hline & $k=1$ & $k=2$ & $k=3$ & $k=4$ & $k=5$ \\
$i=1$ & 200 & 150 & 50 & 50 & 50 \\
$i=2$ & 100 & 100 & 100 & 100 & 100 \\
$i=3$ & 50 & 50 & 200 & 100 & 100 \\
\hline
\end{tabular}


The structural parameters $\alpha_{i}, \beta_{i}$ defining the structural density in (2.4) are to be estimated. Usually this step depends heavily on the application as well on the knowledge of the situation. Let us illustrate a simple estimation procedure that applies to the typical case of the mortality risk in life insurance. One can set $\theta_{i}=q_{x}$ for a certain age $x$, which is interpreted as un unknown conditional probability of death, given alive at age $x$. As a possible method to estimate $\alpha_{i}, \beta_{i}$, we propose to use the estimate $q_{x}^{*}$ of $q_{x}$ given by the life table as well as an estimate of the uncertainty in the estimation of $q_{x}$. In other words estimate $\alpha_{i}, \beta_{i}$ by solving the moment equations

$$
E\left[q_{x}\right]=\frac{\alpha_{i}}{\beta_{i}}, \quad \operatorname{Var}\left[q_{x}\right]=\frac{\alpha_{i}}{\beta_{i}^{2}},
$$

and using estimators of the mean and variance of $q_{x}$. One can take $q_{x}^{*} \cong E\left[q_{x}\right]$ and a good approximation of the variance of $q_{x}$ is given by

$$
\operatorname{Var}\left[q_{x}\right] \cong \frac{q_{x}^{*}\left(1-q_{x}^{*}\right)}{E_{x}}
$$

where $E_{x}$ is the exposure, that is the number of risk years under observation for the estimation of $q_{x}$ in a life table (e.g. LoNDON (1988), chap. 6.2, p. 115). It follows that

$$
\beta_{i}=\frac{E_{x}}{1-q_{x}^{*}}, \quad \alpha_{i}=\beta_{i} q_{x}^{*}
$$

For the EVK 80 table it is known that the total exposure $\Sigma E_{x}$ for the active ages between 20 and 65 is $470^{\prime} 937$. A rough approximation is thus $E_{x} \approx 10^{\prime} 000$. In our example one has

$$
\begin{array}{ll}
\beta_{1}=\frac{10000}{1-q_{30}}=10005.103, & \alpha_{1}=\beta_{1} q_{30}=5.103, \\
\beta_{2}=\frac{10000}{1-q_{40}}=10011.413, & \alpha_{2}=\beta_{2} q_{40}=11.413, \\
\beta_{3}=\frac{10000}{1-q_{50}}=10034.519, & \alpha_{3}=\beta_{3} q_{50}=34.519,
\end{array}
$$

According to (2.5) the expected value of the aggregate claims is equal to $E[X]=3^{\prime} 973^{\prime} 500$. Since $\alpha_{i} / \beta_{i}=q_{x}$ this is equal to the expected value of aggregate claims evaluated using the life table in the traditional way.

\section{Remarks}

(i) In Section 5 we will assume that the amounts at risk $m_{k}$ are non-negative integers. This is an assumption made in most papers of present-day 
applied risk theory. However in pension insurance negative amounts at risk often occur. In this case special mathematical treatment is needed (see e.g. HURLIMANN (1991) and the relevant references mentioned there).

(ii) Since $\operatorname{Pr}\left(X_{k i}=j \mid \theta_{i}\right)$ and $u_{i}\left(\theta_{i}\right)$ are Poisson, resp. Gamma distributed, it follows that the unconditional probability $\operatorname{Pr}\left(X_{k i}=j\right)$ belongs to the negative binomial distribution.

\section{LINK TO THE COLLECTIVE MODEL OF RISK THEORY}

Before undertaking the Bayesian analysis of the linear multivariate Poisson Gamma Bayesian model, we show that it actually identifies with a Bayesian version of the classical collective model of risk theory.

First of all we derive a simple formula for the likelihood $f(x \mid \theta)$ of an aggregate claims observable

$$
x=\sum_{k=1}^{r} m_{k} \sum_{i=1}^{s} x_{k i}
$$

in the Bayesian set-up of Section 2. Consider the matrix $\boldsymbol{x}=\left(x_{k i}\right)^{T}=$ $\left(\boldsymbol{x}_{1}, \ldots, \boldsymbol{x}_{s}\right)^{T}$ of dimension $s x r$, where $\boldsymbol{x}_{i}=\left(x_{1 i}, \ldots, x_{r i}\right), i=1, \ldots, s$, are row vectors of dimension $r$, and the scalar

$$
x_{i}=\left(\boldsymbol{x} \circ \mathbf{1}_{r}\right)_{i}=\sum_{k=1}^{r} x_{k i},
$$

where $\circ$ denotes matrix multiplication and $\boldsymbol{1}_{r}=(1, \ldots, 1)^{T}$ is a unit vector of dimension $r$.

Let $\boldsymbol{m}=\left(m_{1}, \ldots, m_{r}\right)^{T}$ be the vector of possible claim amounts. The scalar product of vectors is denoted by the bracket $\langle\cdot, \cdot\rangle$. Then the aggregate claims observable $x$ may be indifferently identified as scalar product, sum of scalar products, and sum of scalars:

$$
x=\left\langle\boldsymbol{x} \circ \boldsymbol{m}, \mathbf{1}_{s}\right\rangle=\sum_{i=1}^{s}\left\langle\boldsymbol{x}_{\boldsymbol{i}}{ }^{T}, \boldsymbol{m}\right\rangle=\sum_{i=1}^{s} \sum_{k=1}^{r} x_{k i} m_{k},
$$

where $\mathbf{1}_{s}=(1, \ldots, 1)^{T}$ is a unit vector of dimension $s$. The above notations are also defined for the random variable $X$ instead of $x$ and are used throughout. By assumption one has

$$
\begin{aligned}
f(x \mid \theta) & =\operatorname{Pr}\left(\left\langle\boldsymbol{X} \circ \boldsymbol{m}, \mathbf{1}_{s}\right\rangle=x \mid \theta\right) \\
& =\sum_{\left\langle x \circ \boldsymbol{m}, \mathbf{1}_{s}\right\rangle=x} \operatorname{Pr}\left(X_{k i}=x_{k i}, k=1, \ldots, r, i=1, \ldots, s \mid \theta\right) \\
& =\sum_{\left\langle x \circ m_{m}, \mathbf{s}_{s}\right\rangle=x} \prod_{i=1}^{s} \prod_{k=1}^{r} p\left(x_{k i} \mid \theta_{i}\right),
\end{aligned}
$$


where the sum goes over all non-negative integer solutions $x_{k i}, k=1, \ldots, r$, $i=1, \ldots, s$, to the linear inhomogeneous Diophantine equation $\left\langle\boldsymbol{x} \circ \boldsymbol{m}, \mathbf{1}_{s}\right\rangle=x$, and $p\left(x_{k i} \mid \theta_{i}\right)=\operatorname{Pr}\left(X_{k i}=x_{k i} \mid \theta_{i}\right)$. For later use we set for short

$$
p(\boldsymbol{x} \mid \theta)=\prod_{i=1}^{s} \prod_{k=1}^{r} p\left(x_{k i} \mid \theta_{i}\right)
$$

Therefore the likelihood density is given by

$$
f(x \mid \boldsymbol{\theta})=\sum_{\left\langle\boldsymbol{x} \circ \boldsymbol{m}, \mathbf{1}_{s}\right\rangle=x} p(x \mid \boldsymbol{\theta}) .
$$

To obtain the desired link with classical collective risk theory, let us show the following mathematical result.

\section{Proposition 3.1}

Assume that the stochastic system of aggregate claims $(X, \theta)$ satisfies the model assumptions (I) and (II) of Section 2. Then the likelihood density of aggregate claims is conditional compound Poisson of the form

$$
\begin{aligned}
& f(x \mid \theta)=\sum_{n=0}^{\infty} q(n \mid \theta) h^{* n}(x \mid \theta), \text { with } \\
& q(n \mid \theta)=e^{-\lambda(\theta)} \frac{\lambda(\theta)^{n}}{n !}, \quad \lambda(\theta)=\sum_{i=1}^{s} \theta_{i} n_{i},
\end{aligned}
$$

where $n_{i}=\sum_{k=1}^{r} n_{k i}$ is the number of risk units producing claims with probability $\theta_{i}$,

$$
\begin{aligned}
& h(x \mid \theta)=\left\{\begin{array}{ll}
\frac{\lambda_{k}(\theta)}{\lambda(\theta)}, & \text { if } x=m_{k}, k=1, \ldots, r,
\end{array}, \quad 0,\right. \text { else, } \\
& \lambda_{k}(\theta)=\sum_{i=1}^{s} \theta_{i} n_{k i} .
\end{aligned}
$$

\section{Proof :}

(Gerber (1979), pp. 13-14). Since $X_{k i}$ given $\theta_{i}$ is Poisson distributed with parameter $\theta_{i} n_{k i}$, the conditional moment generating function of the random variable $m_{k} X_{k i}$ is equal to

$$
M_{k i}\left(t, \theta_{i}\right)=E\left[e^{t m_{k} X_{k i}} \mid \theta_{\mathrm{i}}\right]=e^{\theta_{i} n_{k i}\left(e_{k}^{i m}-1\right)}
$$


With the conditional independence assumption of (I) one obtains

$$
\begin{aligned}
M_{x}(t, \theta)=E\left[e^{t X} \mid \theta\right] & =\prod_{i=1}^{s} \prod_{k=1}^{r} M_{k i}\left(t, \theta_{i}\right) \\
& =\prod_{k=1}^{r} e^{\lambda_{k}(\theta)\left(e^{t m} k_{k}-1\right)}=e^{\lambda(\theta)\left(\sum_{k=1}^{r} \frac{\lambda_{k}(\theta)}{\lambda(\theta)} e^{t^{m}}-1\right) .}
\end{aligned}
$$

But this is the conditional moment generating function of a conditional compound Poisson distribution with likelihood (3.7). The result is shown.

The above proof actually identifies the linear multivariate Poisson Gamma Bayesian model with the following compound Poisson Gamma Bayesian collective model of risk theory. One has

$$
X=\sum_{i=1}^{N} Y_{i},
$$

and the following model assumptions are fulfilled:

(I') Conditionally on $\theta$ the random variables $Y_{1}, \ldots, Y_{N}, N$ are independent and the $Y_{k}$ 's are identically distributed, that is $Y_{k}=Y$ for all $k$. The random variable $N$ depends upon $\theta$ through the likelihood density $q(n \mid \theta)$ and $Y$ depends upon $\theta$ through $h(x \mid \theta)$ both defined in (3.7).

(II') The structural densities $u_{i}\left(\theta_{i}\right)$ are Gamma densities given by (2.4).

\section{Research problem}

It has been shown that the Bayesian model (I), (II) identifies with the Bayesian model $\left(\mathrm{I}^{\prime}\right)$, (II'). In general, that is when $p\left(x_{k i} \mid \theta_{i}\right)$ and $q(n \mid \theta)$ are not conditionally Poisson distributed, the models (I), (II) and (I'), (II') will not coincide. It seems true that they will coincide only in the Poisson case.

\section{BAYESIAN ANALYSIS OF THE LINEAR MULTIVARIATE POISSON GAMMA MODEL}

Given statistically independent observations of the claims for the different amounts at risk, Bayesian analysis allows to up-date the aggregate claims model. Taking into account available past information, it is thus possible to make predictions about the "true" aggregate claims model associated to a portfolio of risks.

In the first subsection we derive the ubiquitous parameter posterior density $f(\boldsymbol{\theta} \mid \boldsymbol{D})$ given a data set $\boldsymbol{D}$. It allows to calculate the posterior-to-data expected value $E[g(\boldsymbol{\theta}) \mid \boldsymbol{D}]$ for any function $g(\boldsymbol{\theta})$ of the parameter vector $\boldsymbol{\theta}$. In particular one obtains $E[\boldsymbol{\theta} \mid \boldsymbol{D}]$.

In the second subsection we are interested in predictions about the random variable $Y$ of future aggregate claims in the Bayesian model (I), (II). Given a 
data set $\boldsymbol{D}$, the predictive mean $E[Y \mid D]$ is directly obtained from the formula for $E[\boldsymbol{\theta} \mid \boldsymbol{D}]$, while for the calculation of the predictive density $f(y \mid \boldsymbol{D})$, defined by JEwell (1974), one needs the formula for $f(\theta \mid D)$ obtained in the first subsection.

\subsection{Posterior-to-data parameter estimation}

The observation likelihood density of the Bayesian model (I), (II) has been derived in Section 3, formula (3.5). Let us write

$$
\begin{aligned}
& p(\boldsymbol{x} \mid \theta)=\prod_{i=1}^{s} e^{-o_{i} n_{i}} \theta_{i}^{x_{i}} C\left(\boldsymbol{x}_{i}\right), \quad \text { with } \\
& C\left(\boldsymbol{x}_{i}\right)=\prod_{k=1}^{r} \frac{n_{k i}^{x_{k i}}}{x_{k i} !}
\end{aligned}
$$

Consider now the data likelihood density $f(\boldsymbol{D} \mid \boldsymbol{\theta})$ for an observation data matrix $\boldsymbol{D}=\left(\boldsymbol{x}^{(1)}, \ldots, \boldsymbol{x}^{(n)}\right)$ of dimension $s x(n \cdot r)$ containing $n$ statistically indepedent observation matrices $\boldsymbol{x}^{(j)}=\left(x_{k i}^{(j)}\right)^{T}$ of dimension $s x r$, which represent the claims (same notation as in Section 2 with the additional superscript $(j)$ numbering the observation).

Related quantities of interest are the row vectors $\boldsymbol{x}_{i}^{(j)}=\left(x_{1 i}^{(j)}, \ldots, x_{r i}^{(j)}\right)$ of dimension $r$ and the scalars

$$
x_{i}^{(j)}=\sum_{k=1}^{r} x_{k i}^{(j)}
$$

Consider the vector $\boldsymbol{D}_{i}=\left(\boldsymbol{x}_{i}^{(1)}, \ldots, \boldsymbol{x}_{i}^{(n)}\right)$ of dimension $n \cdot r$ such that $D=\left(D_{1}, \ldots, D_{s}\right)^{T}$. The scalar

$$
T_{i}=\sum_{j=1}^{n} x_{i}^{(j)}, \quad i=1, \ldots, s,
$$

represents the total number of claims with unknown probability of occurrence $\theta_{i}$, and turns out to be a sufficient statistic for the considered model. One obtains

$$
f(\boldsymbol{D} \mid \theta)=\prod_{j=1}^{n} p\left(\boldsymbol{x}^{(j)} \mid \theta\right)=\prod_{i=1}^{s} e^{-n 0_{i} n_{i}} \theta_{i}^{T_{i}} \prod_{j=1}^{n} C\left(\boldsymbol{x}_{i}^{(j)}\right) .
$$

To simplify set

$$
C(\boldsymbol{D})=\prod_{i=1}^{s} C\left(\boldsymbol{D}_{i}\right)=\prod_{i=1}^{s} \prod_{j=1}^{n} C\left(\boldsymbol{x}_{i}^{(j)}\right) .
$$


Then one has

$$
f(\boldsymbol{D} \mid \theta)=C(\boldsymbol{D}) \prod_{i=1}^{s} e^{-n \theta_{i} n_{i}} \theta_{i}^{T_{i}} .
$$

From this representation one gets the data probability density by evaluation of a multiple integral, which separates as follows:

$$
\begin{aligned}
f(\boldsymbol{D})=\iint f(\boldsymbol{D} \mid \theta) u(\theta) d \theta & =C(\boldsymbol{D}) \prod_{i=1}^{s} \int \frac{\beta_{i}^{\alpha_{i}} \theta_{i}^{\alpha_{i}+T_{i}-1}}{\Gamma\left(\alpha_{i}\right)} e^{-\left(\beta_{i}+n n_{i}\right) \theta_{i}} d \theta_{i} \\
& =C(\boldsymbol{D}) \prod_{i=1}^{s} \frac{\Gamma\left(\alpha_{i}+T_{i}\right)}{\Gamma\left(\alpha_{i}\right)} \frac{\beta_{i}^{\alpha_{i}}}{\left(\beta_{i}+n n_{i}\right)^{\alpha_{i}+\tau_{i}}}
\end{aligned}
$$

It follows that the parameter posterior density is given by

$$
\begin{aligned}
& f(\theta \mid \boldsymbol{D})=\frac{f(\boldsymbol{D}) u(\theta)}{f(\boldsymbol{D})}=\prod_{i=1}^{s} \operatorname{Gamma}\left(\theta_{i} ; a_{i}, b_{i}\right), \quad \text { with } \\
& a_{i}=\alpha_{i}+T_{i}, \quad b_{i}=\beta_{i}+n n_{i}, \quad i=1, \ldots, s .
\end{aligned}
$$

One sees that it is of the same form as the structural density with up-dated hyperparameters.

At this stage the posterior-to-data expected value $E[g(\theta) \mid D]$ of any function $g(\theta)$ is obtained by evaluation of the multiple integral

$$
E[g(\boldsymbol{\theta}) \mid \boldsymbol{D}]=\iint g(\boldsymbol{\theta}) f(\boldsymbol{\theta} \mid \boldsymbol{D}) d \boldsymbol{\theta}
$$

In particular one gets

$$
\begin{aligned}
E[\theta \mid \boldsymbol{D}]=\left(\ldots, E_{\theta}\left[\theta_{i} \mid \boldsymbol{D}\right], \ldots\right) & =\left(\ldots, \iint \theta_{i} f(\theta \mid \boldsymbol{D}) d \theta, \ldots\right) \\
& =\left(\ldots, \frac{a_{i}}{b_{i}}, \ldots\right)
\end{aligned}
$$

\subsection{Posterior-to-data predictions}

Future observations of the aggregate claims and related quantities are written with the letter $y$ instead of $x$. Our object of study is now the random variable

$$
Y=\left\langle\boldsymbol{Y} \circ \boldsymbol{m}, \mathbf{1}_{s}\right\rangle=\sum_{k=1}^{r} m_{k} \sum_{i=1}^{s} Y_{k i}
$$

representing future aggregate claims in the Bayesian model (I), (II). Immediate 
and of primary importance is the predictive mean of the aggregate claims. Using (4.12) it is obtained as follows:

$$
\begin{aligned}
E[Y \mid \boldsymbol{D}] & =E_{\theta}[E[Y \mid \theta, \boldsymbol{D}]]=E_{\theta}\left[\sum_{i=1}^{s} \theta_{i} \sum_{k=1}^{r} m_{k} n_{k i} \mid \boldsymbol{D}\right] \\
& =\sum_{i=1}^{s} E_{\theta}\left[\theta_{i} \mid \boldsymbol{D}\right] \sum_{k=1}^{r} m_{k} n_{k i}=\sum_{i=1}^{s} \frac{a_{i}}{b_{i}} \sum_{k=1}^{r} m_{k} n_{k i} .
\end{aligned}
$$

If $\boldsymbol{D}$ is the empty set, corresponding to the situation in which no observation is available about the portfolio of risk, one has

$$
E[Y]=E_{\theta}[E[Y \mid \theta]]=\sum_{i=1}^{s} \frac{\alpha_{i}}{\beta_{i}} \sum_{k=1}^{r} m_{k} n_{k i}
$$

clearly the same expression as given in (2.5). In the case of life insurance this means that the expected value of aggregate claims is evaluated according to the life table when no data experience is available.

If $D$ is a non empty set, one has $n \geq 1$, and using (4.10) the formula (4.14) may be rearranged to yield the following multivariate credibility formula:

$$
E[Y \mid \boldsymbol{D}]=\sum_{i=1}^{s}\left\{\left(1-Z_{i}\right) \cdot E\left[\theta_{i} n_{i}\right]+Z_{i} \frac{T_{i}}{n}\right\} \cdot\left\{\frac{1}{n_{i}} \sum_{k=1}^{r} m_{k} n_{k i}\right\}, \text { where }
$$

$Z_{i}=\frac{n n_{i}}{\beta_{i}+n n_{i}} \quad$ is a credibility factor,

$E\left[\theta_{i} n_{i}\right]=n_{i} \frac{\alpha_{i}}{\beta_{i}} \quad$ is the expected number of claims with unknown probability

$$
\frac{T_{i}}{n} \quad \text { is the observed mean number of claims with unknown }
$$

$\frac{1}{n_{i}} \sum_{k=1}^{r} m_{k} n_{k i} \begin{aligned} & \text { is the mean amount at risk for risk units subject to claims } \\ & \text { with probability } \theta_{i} .\end{aligned}$

To evaluate predictive or exact Bayesian stop-loss premiums, one needs besides the predictive mean also the predictive density $f(y \mid \boldsymbol{D})$ of the future aggregate claims. Due to the simple structure of our model, it is not difficult to obtain an explicit analytical formula for the predictive density. With the future observation of aggregate claims $y=\left\langle\boldsymbol{y} \circ \boldsymbol{m}, \mathbf{1}_{s}\right\rangle$ one has from (3.6) and (4.1)

$$
f(\boldsymbol{y} \mid \theta)=\sum_{\left\langle y \circ \boldsymbol{m}, \mathbf{s}_{s}\right\rangle=y} p(\boldsymbol{y} \mid \theta)=\sum_{\left\langle y \circ \boldsymbol{m}, \mathbf{s}_{s}\right\rangle=y} \prod_{i=1}^{s} C\left(\boldsymbol{y}_{i}\right) e^{-\theta_{i} \boldsymbol{n}_{i}} \theta_{i}^{y_{i}} .
$$


The predictive density, which by the way is known to be the unbiased least-squares estimate of $f\left(y \mid \theta_{T}\right)$ for the true value $\boldsymbol{\theta}_{T}$ of $\boldsymbol{\theta}$ (e.g. JEWELL (1974)), is obtained by evaluating the multiple integral

$$
f(y \mid \boldsymbol{D})=\iint f(y \mid \boldsymbol{\theta}) f(\boldsymbol{\theta} \mid \boldsymbol{D}) d \theta .
$$

Using (4.9) and (4.17) the multiple integral separates as follows:

$$
\begin{aligned}
f(y \mid \boldsymbol{D}) & =\sum_{\left\langle y \circ \boldsymbol{m}, \mathbf{1}_{\mathrm{s}}\right\rangle=y} \prod_{i=1}^{s} C\left(y_{i}\right) \int \frac{b_{i}^{a_{i}} \theta_{i}^{a_{i}+y_{i}-1}}{\Gamma\left(a_{i}\right)} e^{-\left(b_{i}+n_{i}\right) \theta_{i}} d \theta_{i} \\
& =\sum_{\left\langle y \circ \boldsymbol{m}, \mathbf{1}_{s}\right\rangle=y} \prod_{i=1}^{s} C\left(y_{i}\right) \frac{\Gamma\left(a_{i}+y_{i}\right)}{\Gamma\left(a_{i}\right)} \frac{b_{i}^{a_{i}}}{\left(b_{i}+n_{i}\right)^{a_{i}+y_{i}}}
\end{aligned}
$$

Using (4.2) and rearranging one obtains the analytical representation

$$
f(y \mid \boldsymbol{D})=\sum_{\left\langle\boldsymbol{y} \circ \boldsymbol{m}, \mathbf{1}_{\mathbf{s}}\right\rangle=y} \prod_{i=1}^{s} C\left(\boldsymbol{y}_{i}\right) \frac{\Gamma\left(a_{i}+y_{i}\right)}{\Gamma\left(a_{i}\right)}\left(\frac{b_{i}}{b_{i}+n_{i}}\right)^{a_{i}} \prod_{k=1}^{r} \frac{\left(\frac{n_{k i}}{b_{i}+n_{i}}\right)^{y_{k i}}}{y_{k i} !}
$$

\section{A RECURSIVE ALGORITHM FOR PREDICTIVE STOP-LOSS PREMIUMS}

In this section we assume for simplicity that $m_{k}=k, k=1, \ldots, r$, that is $\boldsymbol{m}=(1, \ldots, r)$. Other notations remain the same.

Consider the new random variables

$$
\begin{aligned}
& Y^{i}=\sum_{k=1}^{r} k Y_{k i}, \quad \text { with realizations } \\
& y^{i}=\sum_{k=1}^{r} k y_{k i}, \quad i=1, \ldots, s .
\end{aligned}
$$

The set of all non-negative integer solutions to the Diophantine equation

$$
\left\langle\boldsymbol{y} \circ \boldsymbol{m}, \mathbf{1}_{s}\right\rangle=\sum_{i=1}^{s} \sum_{k=1}^{r} k y_{k i}=y
$$

is in one-to-one correspondence with the set of all non-negative integer solutions to the simultaneous Diophantine equations

$$
\sum_{i=1}^{s} y^{i}=y, \quad\left\langle\boldsymbol{y}_{\boldsymbol{i}}{ }^{\boldsymbol{T}}, \boldsymbol{m}\right\rangle=\sum_{k=1}^{r} k y_{k i}=y^{i}, \quad i=1, \ldots, s .
$$


Moreover each Diophantine equation

$$
\left\langle\boldsymbol{y}_{\boldsymbol{i}}{ }^{\boldsymbol{T}}, \boldsymbol{m}\right\rangle=y^{i}, \quad i=1, \ldots, s,
$$

is in one-to-one correspondence with the infinite number of simultaneous Diophantine equations

$$
\left\langle\boldsymbol{y}_{i}{ }^{\boldsymbol{T}}, \boldsymbol{m}\right\rangle=y^{i}, \quad y_{i}=\sum_{k=1}^{r} y_{k i}=k_{i}, \quad k_{i}=0,1,2, \ldots
$$

Using these correspondences the expression (4.20) for the predictive density can be rearranged as follows

$$
\begin{aligned}
& f(y \mid \boldsymbol{D})=\sum_{\Sigma y^{i}=y} \sum_{k_{1}, \ldots, k_{s}=0}^{\infty} \prod_{i=1}^{s} \frac{\Gamma\left(a_{i}+k_{i}\right)}{\Gamma\left(a_{i}\right)}\left(\frac{b_{i}}{b_{i}+n_{i}}\right)^{a_{i}} \cdot S_{k_{1}, \ldots, k_{s}}\left(y^{1}, \ldots, y^{s}\right) \\
& S_{k_{1}, \ldots, k_{s}}\left(y^{1}, \ldots, y^{s}\right)=\sum_{\substack{\left\langle y_{i}, m\right\rangle=y^{i} \\
y_{i}=k_{i} \\
i=1, \ldots, s}} \prod_{i=1}^{s} \prod_{k=1}^{r} \frac{\left(\frac{n_{k i}}{b_{i}+n_{i}}\right)^{y_{k i}}}{y_{k i} !}
\end{aligned}
$$

Using Poisson likelihoods this last expression can be written as

$$
\begin{aligned}
S_{k_{1}, \ldots, k_{s}}\left(y^{1}, \ldots, y^{s}\right) & =\sum_{\substack{\left\langle y_{i}^{T}, m\right\rangle=y^{i} \\
y_{i}=k_{i} \\
i=1, \ldots, s}} \prod_{i=1}^{s} \frac{n_{i}}{e^{\frac{n_{i}+n_{i}}{b_{i}}}} \prod_{k=1}^{r} \operatorname{Po}\left(y_{k i} ; \frac{n_{k i}}{b_{i}+n_{i}}\right) \\
& =\prod_{i=1}^{s} e^{\frac{n_{i}}{b_{i}+n_{i}}} \cdot\left\{\sum_{\substack{\left.\sum_{i}^{T}, m\right\rangle=y^{i} \\
y_{i}=k_{i} \\
i=1, \ldots, s}} \prod_{k=1}^{r} \operatorname{Po}\left(y_{k i} ; \frac{n_{k i}}{b_{i}+n_{i}}\right)\right\} .
\end{aligned}
$$

Let us show how an expression in curly brackets can be evaluated. Each of these sums defines a function of the form

$$
\begin{aligned}
& g_{k}(y)=\sum_{x \in S_{y, k}} \prod_{j=1}^{r} \operatorname{Po}\left(x_{j} ; \lambda_{j}\right), \quad \lambda_{j}>0 \text { the Poisson parameter, } \\
& S_{y, k}=\left\{x \in S_{y}: \sum_{j=1}^{r} x_{j}=k\right\}, \quad k=0,1, \ldots, y, \\
& S_{y}=\left\{x=\left(x_{1}, \ldots, x_{r}\right): \sum_{j=1}^{r} j x_{j}=y\right\}
\end{aligned}
$$


Applying algorithm 2 in the Appendix, one finds that $g_{k}(y)$ can be computed recursively using the formulas

$$
g_{0}(y)=e^{-\lambda} \cdot \delta(y), \quad \lambda=\sum_{j=1}^{r} \lambda_{j}, \quad k g_{k}(y)=\sum_{i=1}^{k} i\left(c_{i}^{*} g_{k-i}\right)(y)
$$

where the functions $c_{i}(z)$ are defined by

$$
c_{i}(z)=\left\{\begin{array}{c}
c(i, j), \quad \text { if } z=i j, \quad j=1, \ldots, r, \quad i=1,2, \ldots, \\
0, \text { else }
\end{array}\right.
$$

with $c(i, j)$ determined by the recursive relation

$$
i c(i, j) \operatorname{Po}\left(0 ; \lambda_{j}\right)=i \operatorname{Po}\left(i ; \lambda_{j}\right)-\sum_{k=1}^{i-1} k c(k, j) \operatorname{Po}\left(i-k ; \lambda_{j}\right) .
$$

One checks immediately that

$$
c(i, j)=\left\{\begin{array}{cc}
\lambda_{j}, \quad i=1, \quad j=1, \ldots r \\
0, & \text { else. }
\end{array}\right.
$$

Hence one has $c_{i}(z)=0, i>1$, and

$c_{1}(j)=\left\{\begin{array}{c}\lambda_{j}, \quad j=1, \ldots, r \\ 0, \text { else. }\end{array}\right.$

Therefore one obtains the following recursive formula

$$
\begin{aligned}
& g_{0}(y)=e^{-\lambda} \delta(y), \quad g_{k}(0)=0, \\
& k g_{k}(y)=\sum_{j=1}^{\min (r, y)} \lambda_{j} g_{k-1}(y-j), \quad k=1,2, \ldots
\end{aligned}
$$

Apply this result to the recursive evaluation of the sum in (5.5) above. Define functions $g_{k}^{i}(y), i=1, \ldots, s$, recursively as follows:

$$
\begin{aligned}
& g_{0}^{i}(y)=e^{-\frac{n_{i}}{b_{i}+n_{i}} \delta(y), \quad g_{\mathrm{k}}^{\mathrm{i}}(0)=0,} \\
& k g_{k}^{i}(y)=\sum_{j=1}^{\min (r, y)} \frac{n_{j i}}{b_{i}+n_{i}} g_{k-1}^{i}(y-j), \quad k=1,2, \ldots
\end{aligned}
$$

For use in numerical evaluation note that

$$
\begin{array}{ll}
g_{k}^{i}(y)=0, & \text { if } \quad y=0,1, \ldots, k-1 \quad \text { or } \quad y>k \cdot r \\
g_{k}^{i}(y) \neq 0, & \text { if } \quad y=k, k+1, \ldots, k \cdot r .
\end{array}
$$

With (5.11) one obtains the following formula

$$
S_{k_{1}, \ldots, k_{s}}\left(y^{1}, \ldots, y^{s}\right)=\prod_{i=1}^{s} e^{\frac{n_{i}}{b_{i}+n_{i}}} g_{k_{i}}^{i}\left(y^{i}\right) .
$$


Since $g_{k_{i}}^{i}\left(y^{i}\right)=0$ for $k_{i}>y^{i}$, it follows that

$$
f(y \mid \boldsymbol{D})=\sum_{\Sigma y^{i}=y} \prod_{i=1}^{s}\left\{\sum_{k=0}^{y^{i}} \frac{\Gamma\left(a_{i}+k\right)}{\Gamma\left(a_{i}\right)}\left(\frac{b_{i}}{b_{i}+n_{i}}\right)^{a_{i}} e^{\frac{n_{i}}{b_{i}+n_{i}}} g_{k}^{i}\left(y^{j}\right)\right\} .
$$

\section{Remark}

In the special case $s=r=1$, one recovers the well-known negative binomial density

$$
f(y \mid \boldsymbol{D})=\frac{\Gamma(a+y)}{\Gamma(a) y !}\left(\frac{b}{b+n}\right)^{a}\left(\frac{n}{b+n}\right)^{y}, \quad y=0,1,2, \ldots
$$

Using algorithm 1 in the Appendix, it is possible now to evaluate formula (5.14) in a recursive manner as follows. Define

$$
f_{i}(x)=\sum_{k=0}^{x} \frac{\Gamma\left(a_{i}+k\right)}{\Gamma\left(a_{i}\right)}\left(\frac{b_{i}}{b_{i}+n_{i}}\right)^{a_{i}} e^{\frac{n_{i}}{b_{i}+n_{i}}} g_{k}^{i}(x) .
$$

Then one has from (5.14)

$$
f(y \mid \boldsymbol{D})=\sum_{\Sigma} \prod_{y^{i}=y}^{s} f_{i=1}\left(y^{i}\right)
$$

Using algorithm 1 one obtains the following recursive algorithm for the exact evaluation of the predictive density:

$$
\begin{gathered}
f(0 \mid \boldsymbol{D})=\prod_{i=1}^{s}\left(\frac{b_{i}}{b_{i}+n_{i}}\right)^{a_{i}}, \\
y f(y \mid \boldsymbol{D})=\sum_{j=1}^{y} j \cdot\left\{\sum_{k=1}^{s} c(j, k)\right\} \cdot f(y-j \mid \mathbf{D}), \quad y=1,2, \ldots, \\
j c(j, k) f_{k}(0)=j f_{k}(j)-\sum_{i=1}^{j-1} i c(i, k) f_{k}(j-i),
\end{gathered}
$$

the $f_{k}(j)$ 's being themselves recursively computed using formulas (5.11) and (5.15). Note that according to (5.12) only summands for which $g_{k}^{i}(y) \neq 0$ are calculated in (5.15). It is important to remark that the numerical process to evaluate $f(y \mid D)$ involves a three-stage nested recursive scheme. Indeed the functions $g_{k}^{i}(y), c(j, k)$ and $f(y \mid \boldsymbol{D})$ are successively recursively computed. However the computation process needs only finitely many operations. The numerical illustration of the next section is based on a concrete computer implementation of the present algorithm. 
Formulas for the recursive evaluation of predictive stop-loss premiums are now easily obtained. For each non-negative integer $T$ let

$$
S L(T \mid D)=E\left[(Y-T)_{+} \mid D\right]
$$

be the predictive stop-loss premium to the priority $T$. Then one has the recursive relations

$$
\begin{array}{rr}
S L(0 \mid \boldsymbol{D})=E[Y \mid \boldsymbol{D}], & \\
S L(T+1 \mid \boldsymbol{D})=S L(T \mid \boldsymbol{D})-1+F(T \mid \boldsymbol{D}), & T=0,1,2, \ldots, \\
F(0 \mid \boldsymbol{D})=f(0 \mid \boldsymbol{D}), & \\
F(T \mid \boldsymbol{D})=F(T-1 \mid \boldsymbol{D})+f(T \mid \boldsymbol{D}), & T=1,2, \ldots
\end{array}
$$

Note that the predictive mean $E[Y \mid D]$ is computed according to the credibility formula (4.16).

\section{A NUMERICAL EXAMPLE}

The following tables are based on the simple example 2.2. They illustrate several extreme situations of interest.

The needed structural parameters $\alpha_{i}, \beta_{i}, i=1,2,3$, have been estimated in Section 2. Given an $n$-year observation period, the up-dated hyperparameters are

$$
b_{i}=\beta_{i}+n n_{i}, \quad a_{i}=\alpha_{i}+T_{i}, \quad i=1, \ldots, s,
$$

where $T_{i}$ is the number of observed deaths in age class $i$ over $n$ years.

If in the linear multivariate model of Section 2 the $\theta_{i}$ 's are assumed to be known with certainty (e.g. the traditional $q_{x}$ of the life table) and the $X_{k i}$ are independent and Poisson $\left(\theta_{i}\right)$ distributed, one gets the traditional collective model of risk theory, that is the usual compound Poisson approximation of the exact individual model of aggregate claims. In Table 1 we compare this classical model with the no data predictive density obtained by setting $n=0$, $T_{i}=0, i=1,2,3$. Table 2 shows the dependency of the predictive distribution and stop-loss premiums upon claims experience. The time of observation is fixed to $n=5$ years and $T_{i}$ varies. In Table 3 the dependency upon time is illustrated assuming an extreme no claims experience over several periods of observation.

Concerning the displayed figures, note that sometimes, due to rounding effects, the cumulative probability may be one, while the corresponding stop-loss premium may not be zero.

TABLE 1

COMPOUND PoISSON (CPM) vs. NO DATA PREDictive MOdel (DPM)

\begin{tabular}{lcc}
\hline \hline & CPM & DPM \\
\hline Expected value & $3^{\prime} 973^{\prime} 500$ & $3^{\prime} 973^{\prime} 500$ \\
Standard deviation & $2^{\prime} 697^{\prime} 638$ & $2^{\prime} 755^{\prime} 165$ \\
\hline
\end{tabular}




\begin{tabular}{lcrrr}
\hline \multirow{2}{*}{ Aggregate claims } & \multicolumn{2}{c}{ Cumulative probability } & \multicolumn{2}{c}{ Stop-loss premiums } \\
& CPM & DPM & CPM & DPM \\
\hline $10 \Delta$ & 0.7131 & 0.7120 & $680^{\prime} 833$ & $703^{\prime} 125$ \\
$20 \Delta$ & 0.9769 & 0.9743 & $41^{\prime} 324$ & $48^{\prime} 057$ \\
$30 \Delta$ & 0.9993 & 0.9990 & $1^{\prime} 120$ & $1^{\prime} 618$ \\
$40 \Delta$ & 1.0000 & 1.0000 & 16 & 32 \\
\hline
\end{tabular}

TABLE 2

DEPENDENCY ON EXPERIENCE BY FIXED TIME OF OBSERVATION $(n=5)$

\begin{tabular}{|c|c|c|c|c|c|c|}
\hline \multirow{4}{*}{$\begin{array}{l}T_{1} \\
T_{2} \\
T_{3}\end{array}$} & \multicolumn{6}{|c|}{ Claims experience } \\
\hline & 0 & 0 & 1 & 1 & 2 & 2 \\
\hline & 0 & 1 & 2 & 3 & 4 & 4 \\
\hline & 0 & 3 & 5 & 8 & 10 & 14 \\
\hline & \multicolumn{6}{|c|}{ Predictive mean } \\
\hline & $3^{\prime} 180^{\prime} 542$ & $3^{\prime} 437^{\prime} 942$ & $3^{\prime} 673^{\prime} 506$ & $3^{\prime} 930^{\prime} 906$ & $4^{\prime} 166^{\prime} 469$ & $4^{\prime} 429^{\prime} 742$ \\
\hline & \multicolumn{6}{|c|}{ Standard deviation } \\
\hline & $2^{\prime} 454^{\prime} 680$ & $2^{\prime} 553^{\prime} 414$ & $2^{\prime} 637^{\prime} 293$ & $2^{\prime} 729^{\prime} 429$ & $2^{\prime} 808^{\prime} 054$ & 2'897'092 \\
\hline $\begin{array}{l}\text { Aggregate } \\
\text { claims }\end{array}$ & \multicolumn{6}{|c|}{ Predictive cumulative probability } \\
\hline 0 & 0.13568 & 0.11602 & 0.09920 & 0.08483 & 0.07253 & 0.06202 \\
\hline 104 & 0.81224 & 0.78071 & 0.75113 & 0.71777 & 0.68700 & 0.65213 \\
\hline $20 \Delta$ & 0.98971 & 0.98582 & 0.98155 & 0.97584 & 0.96979 & 0.96179 \\
\hline $30 \Delta$ & 0.99976 & 0.99961 & 0.99941 & 0.99911 & 0.99875 & 0.99819 \\
\hline $40 \triangle$ & 1.00000 & 0.99999 & 0.99999 & 0.99998 & 0.99997 & 0.99995 \\
\hline $\begin{array}{l}\text { Aggregate } \\
\text { claims }\end{array}$ & \multicolumn{6}{|c|}{ Predictive stop-loss premiums } \\
\hline 0 & $3^{\prime} 180^{\prime} 512$ & $3^{\prime} 437^{\prime} 942$ & $3^{\prime} 673^{\prime} 506$ & $3^{\prime} 930^{\prime} 906$ & $4^{\prime} 166^{\prime} 469$ & $4^{\prime} 429^{\prime} 742$ \\
\hline $10 \Delta$ & $394^{\prime} 778$ & $483^{\prime} 804$ & $572^{\prime} 673$ & $680^{\prime} 274$ & $785^{\prime} 832$ & $914^{\prime} 391$ \\
\hline $20 \Delta$ & $17^{\prime} 059$ & $24^{\prime} 405$ & $32^{\prime} 805$ & $44^{\prime} 538$ & $57^{\prime} 477$ & $75^{\prime} 378$ \\
\hline $30 \mathrm{~A}$ & 352 & 590 & 904 & $1^{\prime} 409$ & $2^{\prime} 041$ & $3^{\prime} 037$ \\
\hline $40 \Delta$ & 4 & 8 & 14 & 26 & 42 & 71 \\
\hline
\end{tabular}

TABLE 3

DEPENDENCY ON TIME OF OBSERVATION FOR NO CLAIMS EXPERIENCE

\begin{tabular}{lcccccc}
\hline \multicolumn{7}{c}{ Observation period } \\
\hline$n$ & 1 & 2 & 3 & 4 & 5 & 10 \\
\hline \multicolumn{7}{c}{ Credibility factors } \\
\hline$Z_{1}$ & 0.04760 & 0.09087 & 0.13038 & 0.16660 & 0.19992 & 0.33322 \\
$Z_{2}$ & 0.04757 & 0.09081 & 0.13031 & 0.16651 & 0.19982 & 0.33308 \\
& 0.04746 & 0.09062 & 0.13004 & 0.16619 & 0.19945 & 0.33257 \\
\hline
\end{tabular}


Predictive mean

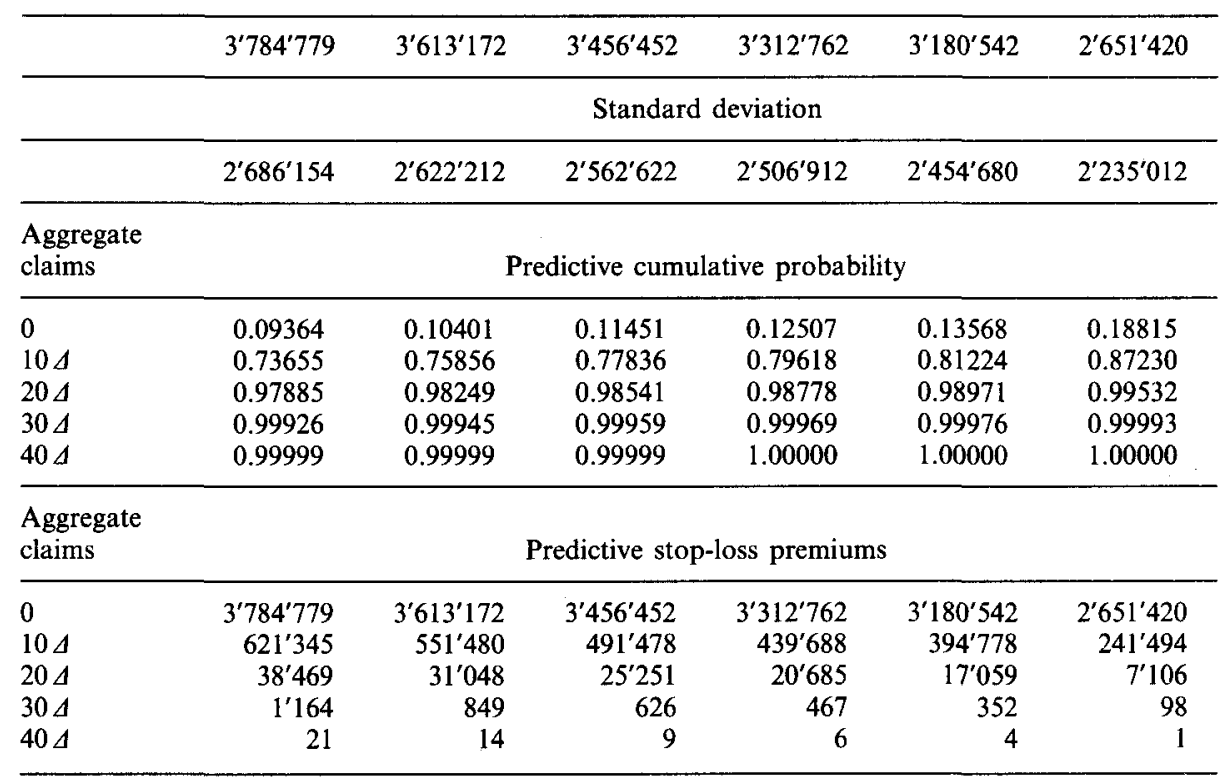

\section{APPENDIX}

In order to be self-contained, as well as for the convenience of the reader, the main results of HÜRLIMANN (1990a) are reproduced without proof.

Let $X_{j}, j=1,2, \ldots$, be mutually independent random variables taking values in the non-negative integers. Let $f_{i}(i)=\operatorname{Pr}\left(X_{j}=i\right)$ and assume that $f_{j}(0)>0$. Consider a linear combination of random variables

$$
Y=\sum_{k=1}^{\infty} a_{k} X_{k}, \quad a_{k} \in N_{0} .
$$

Assume that $a_{1} \geq a_{2} \geq \ldots \geq a_{k}>0, k=1,2, \ldots$ Given the $a_{k}$ 's and the $f_{j}(i)$ 's, the following two-stage nested recursive algorithm for the exact computation of $f(y)=\operatorname{Pr}(Y=y)$ is available (Theorem 1 in HürLIMANN (1990a)).

\section{Algorithm 1}

Under the above conditions one has

$$
f(0)=\prod_{j \in I_{\infty}} f_{j}(0)
$$


If $y>0$ then one has

$$
\begin{aligned}
y f(y) & =\sum_{k \in I_{y}} a_{k} \sum_{s=1}^{\left[y / a_{k}\right]} s c(s, k) f\left(y-s a_{k}\right) \\
& =\sum_{s=1}^{y} s \sum_{k \in I_{[y / s]}} a_{k} c(s, k) f\left(y-s a_{k}\right) .
\end{aligned}
$$

In these formulas

$$
I_{y}=\left\{k: 0<a_{k} \leq y\right\},
$$

$$
I_{\infty}=\left\{\mathbf{k}: a_{k}>0\right\},
$$

(A.7)

$[y]$ is the greatest integer contained in $y$,

and $c(s, k)$ is determined by the recursive relation

$$
s c(s, k) f_{k}(0)=s f_{k}(s)-\sum_{j=1}^{s-1} j c(j, k) f_{k}(s-j) .
$$

The apparent complexity of Algorithm 1 is reduced by abstraction as follows. For each $s=1,2, \ldots$, define

$$
c_{s}(z)=\left\{\begin{array}{c}
c(s, k), \quad \text { if } z=s a_{k} \text { for some } k, \\
0, \quad \text { otherwise. }
\end{array}\right.
$$

Note the index notation error in HÜRIMANN (1990a), where the indices $s, k$ must be exchanged in order to yield correct practical results. With the change of variable $z=s a_{k}$ the relation (A.4) can be rewritten as

$$
y f(y)=\sum_{z=1}^{y} z \sum_{s=1}^{\infty} c_{s}(z) f(y-z) .
$$

Then set $\lambda=-\ln \{f(0)\}$ and define the function

$$
h(z)=\frac{1}{\lambda} \sum_{s=1}^{\infty} c_{s}(z)
$$

Then Algorithm 1 is equivalent to the simple Panjer-like recursion

$$
\begin{gathered}
f(0)=e^{-\lambda}, \\
y f(y)=\lambda \sum_{z=1}^{y} z h(z) f(y-z) .
\end{gathered}
$$

The second important computational result contained in HúrLIMANN (1990a), theorem 2, concerns an alternative recursive procedure to evaluate 
$f(y)$. For this one considers for each $y \in N_{0}$ the Diophantine set

$$
\text { (A.13) } S_{y}=\left\{\boldsymbol{x}=\left(0, \ldots, 0, x_{k_{y}+1}, x_{k_{y}+2}, \ldots, x_{k_{0}}, x_{k_{0}+1}, \ldots\right): x_{k} \in N_{0}, \sum_{k \in I_{y}} a_{k} x_{k}=y\right\}
$$

consisting of all non-negative integer solutions of the linear equation

$$
\sum_{k=1}^{\infty} a_{k} x_{k}=y
$$

In this definition

$$
k_{x}=\left\{\begin{array}{c}
\max \left\{k: a_{k}>x\right\}, \text { if } x<a_{1}, \\
0, \text { if } x \geq a_{1} .
\end{array}\right.
$$

and it follows that

$$
I_{y}=\left\{k_{y}+1, k_{y}+2, \ldots, k_{0}\right\} .
$$

From the independence of the $X_{j}^{\prime}$ 's, one deduces that

$$
f(y)=c_{y} \sum_{x \in S_{y}} \prod_{j \in I_{y}} f_{j}\left(x_{j}\right), \quad c_{y}=\prod_{j=1}^{k_{y}} f_{j}(0) .
$$

Consider the following subsets of $S_{y}$ :

$$
S_{y, k}=\left\{x \in S_{y}: \sum_{j \in I_{y}} x_{j}=k\right\}, \quad k=0,1, \ldots, y,
$$

and define functions

$$
g_{k}(y)=c_{y} \sum_{x \in S_{y, k}} \prod_{j \in I_{y}} f_{j}\left(x_{j}\right), \quad k \in N_{0},
$$

with the convention that $g_{k}(y)=0$ whenever the sum is empty. From (A.14) one sees that

$$
f(y)=\sum_{k=0}^{\infty} g_{k}(y) .
$$

Algorithm 2 shows how to compute $f(y)$ by the successive recursive evaluation of $g_{k}(y)$ using the $c_{k}(y)$ 's defined in (A.9).

\section{Algorithm 2}

One has the following recursive formula

$$
\begin{gathered}
g_{0}(y)=f(0) \delta(y), \\
k g_{k}(y)=\sum_{j=1}^{k} j\left(c_{j}^{*} g_{k-j}\right)(y) .
\end{gathered}
$$


It is important to observe that Algorithm 2 is a Panjer-like recursion in the space of discrete distributions with addition and convolution as operations. It is worthwile to mention that Algorithm 2 generalizes the results obtained through the shovelboard approach of VAN KLINKEN (1960) recently revisited by Alting von Geusau (1990).

\section{ACKNOWLEDGEMENT}

I am very grateful to the referees for their detailed comments and helpful suggestions for a better presentation.

\section{REFERENCES}

Alting von Geusau, B. (1990) The shovelboard approach revisited. XXII ASTIN Colloquium, Montreux.

AMmeter, H. (1948) A generalization of the collective theory of risk in regard to fluctuating basic probabilities. Scandinavian Actuarial Journal.

Ammeter, H. (1949) Die Elemente der kollektiven Risikotheorie von festen und zufallsartig schwankenden Grundwahrscheinlichkeiten. Mitteilungen der Vereinigung Schweiz. Vers. math., 35-95.

Beard, R.E., Pentikäinen, T. and Pesonen, E. (1984) Risk Theory, the stochastic basis of insurance, third edition. Chapman and Hall.

Bertram, J. and Feilmeier, M. (1987). Anwendung numerischer Methoden in der Risikotheorie. Schriftenreihe Angewandte Versicherungsmathematik, Heft 16. Verlag Versicherungswirtschaft, Karlsruhe.

Gerber, H.U. (1979) An introduction to mathematical Risk Theory. Huebner Foundation for Insurance Education. University of Pennsylvania.

HÚrLimanN, W. (1990a) On linear combinations of random variables and Risk Theory. In: Methods of Operations Research 63, XIV Symposium on Operations Research, Ulm, 1989, $11-20$

HUrlimann, W. (1990b) Pseudo compound Poisson distributions in Risk Theory. ASTIN Bulletin 20, 57-79.

Húrlimann, W. (1991) Negative claim amounts, Bessel functions, Linear Programming and Miller's algorithm. Insurance: Mathematics and Economics 10, 9-20.

JEWELL, W.S. (1974) The credible distribution. ASTIN Bulletin 7, 237-269.

JewELL, W. S. (1986) Introduction to Bayesian Statistics and Credibility Theory. Operations Research Center, University of California, Berkeley, presented at the Summer School of the Association of Swiss Actuaries, Gwatt, Switzerland.

JEWELL, W.S. (1974) Up the misty staircase with Credibility Theory. Mitteilungen der Vereinigung Schweiz. Vers. math., 281-312.

DE JoNG, P. (1983). The mean square error of a randomly discounted sequence of uncertain payments. In: De Vylder, F., Goovaerts, M., Haezendonck, J. Premium Calculation in Insurance, NATO ASI Series, Series C: Mathematical and Physical Sciences, vol. 121, 449-459.

VAN Klinken, J. (1960) Actuariële Statistiek, Syllabus at the Institute of Actuarial Sciences and Econometrics of the University of Amsterdamm, pp. 13-15 (In Dutch).

Klugman, S. A. (1989) Bayesian modelling of mortality catastrophes. Insurance: Mathematics and Economics 8, $159-164$.

LONDON, D. (1988) Survival Models and their Estimation, second edition. ACTEX Publications, Winsted and New Britain, Connecticut.

NorberG, R. (1987) A note on experience rating of large group life contracts. Mitteilungen der Vereinigung Schweiz. Vers. math., 17-34.

PANJER, H.H. (1981) Recursive evaluation of a family of compound distributions. ASTIN Bulletin $12,22-26$. 
De PrIL, N. (1986) On the exact computation of the aggregate claims distribution in the individual life model. ASTIN Bulletin 16, 109-112.

DE PrIL, N. (1989) The aggregate claims distribution in the individual model with arbitrary positive claims. ASTIN Bulletin 19, 9-24.

Wolthuis, H. and VAN HoEk, I. (1986) Stochastic models for Life Contingencies. Insurance: Mathematics and Economics 5, 217-254.

WERNER HÜrLIMANN

Allgemeine Mathematik, Winterthur-Leben, Römerstrasse 17, CH-8401 Winterthur. 